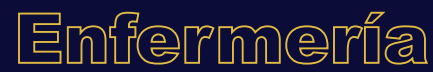

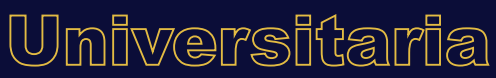

\section{Validez y confiabilidad de la Escala de Adopción al Rol Materno en madres adolescentes mexicanas}

\section{Validity and reliability of the Maternal Role Adoption Scale among mexican teenage mothers}

\section{Validade e confiabilidade da Escala de Adoção do Rolo Materno em mães adolescentes mexicanas}

\section{M.L. Santos-Diaz ${ }^{\mathrm{a} 1}$, D.M. Pérez-Calderón ${ }^{\mathrm{b}}{ }^{\text {, }}$ E. Lozada-Perezmitre ${ }^{\mathrm{c} 2}, \mathbf{N}$. Ramírez-Girón ${ }^{\mathrm{d} 3}$, E. Landeros-Olvera ${ }^{\mathrm{e}{ }^{*}}$}

ORCID

a $\underline{0000-0003-2202-4874}$

d $\underline{0000-0002-8312-6287}$

b $0000-0003-0401-2722$

e $0000-0001-6270-1759$

c0000-0002-0515-8662

${ }^{1}$ Secretaría de Salud Puebla, Hospital de Traumatología y Ortopedia

${ }^{2}$ Benemérita Universidad Autónoma de Puebla, Facultad de Enfermería, Puebla, México

3 Universidad de las Américas Puebla, Programa de Enfermería, Puebla, México

Recibido: 28 abril 2020

Aceptado: 13 mayo 2021

RESUMEN

Introducción: El nivel de Adopción del Rol Materno (ARM) en madres adolescentes es esencial, dado que durante esta etapa las funciones de responsabilidad y decisión no están completamente desarrolladas y pueden afectar el cuidado del bebé. La teoría de la ARM de Ramona Mercer fundamenta este trabajo.

Objetivo: Adaptar la Escala de ARM de Garrido y Marchan para probar la validez y confiabilidad en adolescentes mexicanas.

* Autor para correspondencia. Correo electrónico: dr.erick.landeros@gmail.com https://doi.org/10.22201/eneo.23958421e.2021.1.880

1665-7063/@ 2021 Universidad Nacional Autónoma de México, Escuela Nacional de Enfermería y Obstetricia. Este es un artículo Open Access bajo la licencia CC BY-NC-ND (http://creativecommons.org/licenses/by-nc-nd/4.o/). 
Métodos: Análisis estadístico descriptivo e inferencial. Diseño secuencial exploratorio, se realizó adecuación semántica; validación con 10 jueces expertos; prueba piloto realizada en 30 madres adolescentes; la versión final del instrumento se aplicó en 90 madres entre 1019 años de edad. El anonimato, la confidencialidad y el consentimiento informado fueron considerados.

Resultados: Las palabras dar de lactar se reemplazó por dar pecho. Mediante el análisis por jueces se obtuvo el Índice de Validez por Ítem: 7; Criterio de Validez: 12.9\%; Índice de Validez de Contenido: 8.7. En la prueba piloto no hubo cambios. En la prueba utilizando la versión final del instrumento, madres con edad promedio de 17.2 \pm 1.6 , el alpha de Cronbach fue 0.824 Discusión: La versión validada de la escala muestra ser sólida en el contexto mexicano y refuerza la escala original dada la adición de los puntos de corte para identificar el nivel de ARM en mujeres adolescentes durante el puerperio inmediato.

Conclusiones: La Escala de ARM de Garrido y Marchan es válida y confiable en madres adolescentes mexicanas, esta herramienta puede fortalecer las intervenciones de enfermería para mejorar resultados de salud.

Palabras claves: Rol materno; confiabilidad; validez; conducta materna; adolescente; México.

\section{ABSTRACT}

Introduction: A high level of Adoption of the Maternal Role (AMR) among teenage mothers is essential because, during adolescence, the behaviors of responsibility and decision are not fully developed and thus, the care of the baby could be compromised. The AMR Theory of Ramona Mercer is the framework for the present study.

Objective: To use the AMR scale of Garrido and Marchan among mexican adolescent mothers in order to test its validity and reliability.

Methods: This is a descriptive and inferential study with sequential and exploratory design and with semantic adequation. Ten expert judges were included in the validation process. A pilot test was developed and tested on 30 mexican adolescent mothers. The final version of the instrument was given to 90 mothers with ages ranging from 10 to 19 years old. Anonymity and confidentiality were guaranteed; and the informed consent from all the participants was obtained.

Results: The word milk-feeding was replaced by breastfeeding. From the expert judges' analysis, an Item Validity Index of 7, a Validity Criterion of $12.9 \%$, and a Validity of Context Index of 8.7 were obtained. No changes were made on the test pilot structure. The final version of the instrument showed a Cronbach Alpha of .824. The average age of the participants was $17.2 \pm 1.6$.

Discussion: The validated version of the scale demonstrates consistency for the context of Mexico. Therefore, this scale can be used to identify the level of Adoption of the Maternal Role among mexican adolescent mothers.

Conclusions: The AMR Scale of Garrido and Marchan is valid and reliable to be used in mexican teenage mothers. Moreover, this tool can help strengthen the nursing interventions aimed at improving the health results of this population group.

Keywords: Maternal role; reliability; validity; maternal behavior; adolescent; Mexico. 


\section{RESUMO}

Introdução: O nível de Adoção do Rolo Materno (ARM) em mães adolescentes é essencial, dado que durante esta fase as funções de responsabilidade e decisão não são completamente desenvolvidas e podem afetar o cuidado do bebê. A teoria da ARM de Ramona Mercer fundamenta este trabalho.

Objetivo: Adaptar a Escala de ARM de Garrido e Marchan para testar a validade e confiabilidade em adolescentes mexicanas.

Métodos: Análise estatística descritiva e inferencial. Desenho sequencial exploratório, realizou-se adequação semântica; validação com 10 juízes especialistas; prova piloto realizada em 30 mães adolescentes; a versão final do instrumento foi aplicada a 90 mães entre 1019 anos de idade. Foram considerados o anonimato, a confidencialidade e o consentimento informado.

Resultados: As palavras dar a lactar foram substituídas por amamentar. Mediante a análise por juízes obteve-se o Índice de Validade por Item: 7; Critério de Validade: 12.9\%; Índice de Validade de Conteúdo: 8.7. Na prova piloto não houve mudanças. Na prova utilizou-se a versão final do instrumento, as mães com média de idade de $17.2 \pm 1.6$, o alpha de Cronbach foi 0.824 .

Discussão: A versão validada da escala mostra ser sólida no contexto mexicano e reforça a escala original dada pelo acréscimo dos pontos de corte para identificar o nível de ARM em mulheres adolescentes durante no puerpério imediato.

Conclusões: A escala Garrido e Marchan MRA é válida e confiável em mães adolescentes mexicanas, esta ferramenta pode fortalecer as intervenções de enfermagem para melhorar resultados de saúde.

Palavras chave: Papel materno; confiabilidade; validade; comportamento materno; adolescente; México.

\section{INTRODUCCIÓN}

De acuerdo con la Organización para la Cooperación y el Desarrollo Económico (OCDE) y el Banco Mundial'2 ${ }^{2}$ América Latina y el Caribe presentan las mayores tasas de embarazo en adolescentes, se calcula que hay 72 nacimientos por cada 1000 mujeres entre 15 y 19 años de edad. El Fondo de las Naciones Unidas para la Infancia (UNICEF) 3 afirma que el número de madres adolescentes menores de 15 años ha aumentado 22\% en estas zonas. México ocupa el primer lugar en embarazos adolescentes entre los países de la OCDE, pues aproximadamente ocurren al año 340 mil nacimientos de mujeres menores de 19 años con una tasa de fecundidad de 77 nacimientos por cada mil adolescentes de 15 a 19 años de edad4.

Más allá de los problemas convencionales que preocupan al sistema de salud como la morbimortalidad en este grupo de mujeres, los programas de atención en salud no enfatizan el cuidado de las adolescentes y una vez que se convierten en madres deben adoptar el nuevo rol. Por consiguiente, es vital intervenir en esta etapa del desarrollo femenino dado que el ser madre afecta el proyecto de vida de cada adolescente y pone en relieve no sólo cambios biológicos, sino también psicológicos y sociales, ocasionando inestabilidad al ser responsable de un bebé, sobre todo, cuando las funciones de responsabilidad y toma de decisiones no están desarrolladas totalmente ${ }^{5}$. Además, es importante contar con herramientas que faciliten las intervenciones de los profesionales de salud, quienes 
poseen las competencias necesarias para mejorar el proceso de Adopción del Rol Materno (ARM) en esta etapa de la vida.

La teoría de Ramona Mercer ${ }^{6}$ define la ARM como un proceso interactivo entre la madre con su hijo dando origen a un vínculo afectivo. La teoría propone tres constructos con diferentes dimensiones: A. Microsistema (rol de madre, estimulación del bebé, bienestar, afecto, aceptación, preocupación y protección del bebé); B. Mesosistema (interacción con la pareja respecto al bebé, interacción con la familia respecto al bebé); C. Macrosistema (cuidados del bebé, conocimiento y cultura relacionados con el bebé).

Se han creado diferentes instrumentos que miden aspectos relacionados como la autoeficacia en el desempeño del rol materno ${ }^{7}$ y el apego entre padres e hijos ${ }^{8,9}$; sin embargo, son escasos los instrumentos que evalúan la ARM desde la visión teórica de Mercer en contextos de habla hispana ${ }^{10,11}$. La revisión de la literatura reportó la creación y validez de la Escala de Adopción del Rol Materno (E-ARM) de Garrido y Marchán ${ }^{12}$ en población adolescente peruana; posteriormente, Vargas et al. ${ }^{10}$ reportaron la adaptación cultural de la misma escala en Colombia.

Ahora bien, para el caso de México existe la necesidad de contar con un instrumento confiable en madres adolescentes para medir el impacto de las intervenciones de salud que promueven la ARM, de ahí la importancia para validar la E-ARM de Garrido y Marchán ${ }^{12}$. Con base en lo anterior, el objetivo de este estudio fue adaptar la E-ARM para probar la validez y confiabilidad en adolescentes mexicanas.

\section{MÉTODOS}

Diseño secuencial cuantitativo transversal en el cual se llevaron a cabo diferentes etapas para alcanzar el objetivo propuesto ${ }^{13,14}$.

- Primera etapa. Adecuación de la E-ARM ${ }^{12}$. Consistió en modificar la semántica y redacción de los ítems al contexto sociocultural mexicano, se respetó orden y significado de cada ítem ${ }^{15}$.

- Segunda etapa. Validación por técnica de jueces. Las adecuaciones realizadas en la primera etapa fueron sometidas a una evaluación cuanti-cualitativa con base en la metodología de Waltz et al. ${ }^{13}$ para obtener la validez de contenido ${ }^{14,16,17}$. Se seleccionaron diez profesionales de enfermería expertos en materno infantil y obstetricia con grado académico de maestría y doctorado. Para el análisis cuantitativo se valoró a cada ítem la relación con la definición y las dimensiones de ARM de acuerdo a la siguiente escala: $0=$ Definitivamente no está relacionado; $1=$ No relacionado; $2=$ No seguro de su relación, los reactivos requieren más revisión; $3=$ Relacionado, pero es necesario realizar pequeñas modificaciones y $4=$ =xtremadamente relacionado, sin alteración. Para la evaluación, se consideraron dos opciones para cada ítem, Aceptable (IA) cuando obtuvo un puntaje igual o mayor a tres, y No Aceptable (INA) con un puntaje menor a tres. Con el número total de IA e INA se realizó el análisis de contenido ${ }^{13,16,17}$. Para este análisis se calcula el Índice de Validez por Ítem (IVXI), adecuado con un índice igual o mayor a siete, el Criterio de Validez (CV) que indica la posibilidad de error tipo II (aceptable hasta 20\%) y el Índice de Validez de Contenido (IVC), favorable cuando se acerca a la decena ${ }^{13,16,17 .}$

- Tercera etapa. Prueba piloto. A partir de la segunda versión de la E-ARM se realizó un pilotaje con 30 madres adolescentes en una institución de salud pública, se incluyeron adolescentes de 10 a 19 años de edad, primigestas, que supieran leer y escribir, antecedente de recién evento obstétrico quirúrgico o fisiológico, con producto de la concepción vivo, sin complicaciones médicas en la madre o el recién nacido. El objetivo fue probar la factibilidad de procedimientos, 
condiciones de aplicación y claridad de los ítems para identificar y eliminar posibles variables confusoras.

- Cuarta etapa. Aplicación de la versión final de la escala. Esta etapa fue probada en un grupo de adolescentes entre 10 y 19 años, en puerperio derivado de un parto eutócico o cesárea, en compañía de pareja o algún miembro de la familia, con producto de la concepción vivo, sin complicaciones médicas en la madre o el recién nacido. El muestreo fue no probabilístico, la muestra fue calculada mediante análisis de potencia, considerando un alpha de Cronbach 0.05, tamaño de efecto 0.50, poder de 0.90 con un efecto de atrición de 7\%, por lo que se obtuvo una $n=90$ participantes ${ }^{13,16,17}$. La recolección de los datos se realizó durante tres meses en un hospital público de segundo nivel especializado en la mujer.

Instrumento: Se utilizó el instrumento de Garrido y Marchán ${ }^{12}$ en su versión para población mexicana, la cual respeta las mismas características de la estructura original, consta de 56 reactivos que evalúan tres constructos identificados en la Teoría de Adopción del Rol Materno ${ }^{6}$, se distribuyen en 10 dimensiones y se desglosan de la siguiente manera:

A. Microsistema: Aceptación del bebé (ítems 1-3, 5, 9, 16, 25, 31, 33, 42); Contacto con el rol de madre (ítems 8, 26, 27, 48, 49, 50); Estimulación del bebé (ítems 11, 13, 14, 30, 38, 41, 53); Expresiones maternales de afecto hacia el bebé (ítems 18-21, 29); Bienestar del bebé (ítems 6, 15, 23, 51, 56); Preocupación y protección del bebé (ítems 24, 35, 43, 44, 47).

B. Mesosistema: Interacción con la pareja respecto al bebé (ítems 12, 28, 40, 52, 54); Interacción con la familia de origen respecto al bebé (ítems 10, 36, 45, 46, 55).

C. Macrosistema: Cuidados del bebé (ítems 4, 7, 22, 34); Conocimiento y cultura relacionados con el bebé (ítems 17, 32, 37, 39).

El formato de respuesta es con base en una escala tipo Likert con los siguientes valores: 1=Totalmente en Desacuerdo (TD); $2=$ Desacuerdo (D); $3=$ De Acuerdo (A) y $4=$ Totalmente de Acuerdo (TA). El puntaje mínimo es 56 y el máximo es 224. El nivel de adopción del rol materno puede ser bajo ( 56 a 180 puntos), medio (181 a 202 puntos) o alto (203 a 224 puntos). El instrumento presenta un alpha de Cronbach aceptable.

Plan de análisis estadístico: Las variables cuantitativas se presentan con medias y desviaciones estándar (DE), las categóricas con frecuencias y porcentajes. Para determinar la confiabilidad de la escala se utilizó la prueba alpha de Cronbach, la normalidad de las variables se calculó a través de la prueba de bondad y ajuste de Kolmogorov-Smirnov. Se estableció la validez de la escala mediante un Análisis Factorial Exploratorio (AFE) a partir del método de extracción de componentes principales con el cálculo del índice de Kaiser-Meyer-Olkin (KMO) y el test de esfericidad de Bartlett. Para mejorar la comprensión en la extracción de los datos y diferencia de cargas factoriales para cada reactivo se realizó la rotación varimax. En todos los análisis estadísticos se utilizó el paquete SPSS versión 21.

Con el fin de mejorar la comprensión de los resultados del instrumento de ARM, la variable se transformó a índices de cero a cien y se ajustaron los siguientes puntos de corte: 0 a 24 ARM muy bajo; 25 a 49 ARM bajo; 50 a 74 ARM alto; 75 a 100 ARM muy alto. Los puntos de corte se construyeron con base en el percentil 25 que sugiere la literatura (Waltz et al.) (13 $^{13}$ se propusieron para facilitar la comprensión del nivel de ARM desde el punto de vista estadístico. Además, para mantener el sentido de la puntuación, los ítems 1, 7, 8, 16, 22, 29, 37, 48-50, se invirtieron de acuerdo con la publicación original. 
Consideraciones éticas: La presente investigación se realizó de acuerdo con las disposiciones de la Ley General de Salud en Materia de Investigación. Esta investigación se consideró sin riesgo ya que no existió ninguna intervención o modificación intencionada de las variables, se realizó considerando el respeto a la dignidad del ser humano como sujeto de investigación, se contó con el consentimiento informado mediante el cual la madre adolescente autorizó la participación en la investigación -en el caso de las menores de edad su representante legal fue quien lo autorizó-, con la capacidad de libre elección y sin coacción alguna.

Conforme al Capítulo IV correspondiente a la investigación en mujeres en edad fértil, embarazadas, durante el trabajo de parto, puerperio, lactancia y recién nacidos, se cumplió con lo estipulado en el Artículo 50 que enfatiza la posibilidad de realizar investigaciones en mujeres en puerperio siempre y cuando no interfieran con la salud del binomio. El presente trabajo fue avalado por la Secretaría de Investigación y Estudios de Posgrado de una universidad pública con número de registro: $177 / 2015$.

\section{RESULTADOS}

Primera etapa. Se decidió a priori que las palabras lactancia, dar(le) de lactar, serían sustituidas por las palabras pecho, dar(le) pecho, también se agregaron algunos signos gramaticales (coma) ${ }^{18}$.

Segunda etapa. En el análisis cuantitativo la mayoría de los ítems se consideraron aceptables. El IVXI se encontró adecuado, el CV representa 12.9\% de posibilidad de error (aceptable) y el IVC es cercano a la decena (favorable) tal como se muestra en la tabla 1.

\begin{tabular}{|c|c|c|c|c|c|c|}
\hline Jueces & $\begin{array}{c}\text { Ítems } \\
\text { No Aceptables } \\
\text { (INA) }\end{array}$ & $\begin{array}{c}\text { Ítems } \\
\text { Aceptables } \\
\text { (IA) }\end{array}$ & Variable & Fórmula & Sustitución & Resultado \\
\hline 1 & 26 & 28 & \multirow[t]{3}{*}{ IVXI } & \multirow{3}{*}{$\begin{array}{l}\text { Total de INA/ } \\
\text { Número de } \\
\text { jueces }\end{array}$} & \multirow[t]{3}{*}{$70 / 10$} & \multirow[t]{3}{*}{7} \\
\hline 2 & 4 & 50 & & & & \\
\hline 3 & 10 & 44 & & & & \\
\hline 4 & 2 & 52 & \multirow[t]{4}{*}{$\mathrm{CV}$} & \multirow{4}{*}{$\begin{array}{c}\text { (IVXI/Total de } \\
\text { ítems)100 }\end{array}$} & \multirow[t]{4}{*}{$(7 / 54) 100$} & \multirow[t]{4}{*}{12.9} \\
\hline 5 & 6 & 48 & & & & \\
\hline 6 & 2 & 52 & & & & \\
\hline 7 & 8 & 46 & & & & \\
\hline 8 & 5 & 49 & \multirow[t]{3}{*}{ IVC } & \multirow{3}{*}{$\begin{array}{l}\text { IA/Total de } \\
\text { ítems }\end{array}$} & \multirow[t]{3}{*}{$470 / 54$} & \multirow[t]{3}{*}{8.7} \\
\hline 9 & 3 & 51 & & & & \\
\hline 10 & 4 & 50 & & & & \\
\hline
\end{tabular}

Variables: IVXI (Índice de Validez por Ítem), CV (Criterio de Validez), IVC (Índice de Validez de Contenido)

Para la fase cualitativa, de los 11 ítems que se modificaron en la versión original, cuatro de ellos requirieron nuevamente modificaciones derivado de las observaciones cualitativas de los jueces (ítems 1, 15, 25, 42). En un segundo análisis se necesitó modificar 14 ítems (3, 5, 12, 21, 28, 31, 34, 35, 40, $41,47,48,52$ y 54). En total fueron 25 ítems modificados de la escala original (45.4\%), con los cuales se obtuvo la segunda versión del instrumento. 
Tercera etapa. En la aplicación de la E-ARM en la prueba piloto no fue necesario realizar cambios a los ítems.

Cuarta etapa. La E-ARM se aplicó en 90 adolescentes, edad de $17.2 \pm 1.6$ años; la vía de nacimiento fue cesárea en $51.1 \%$ de los casos. 70\% de las adolescentes se encuentran casadas o en unión libre y más del 90\% no tenían trabajo remunerado. 27.8\% cuenta con educación básica (primaria y secundaria), $16.7 \%$ media superior, 3.3\% estudios de nivel superior y 52.2\% tenía estudios incompletos.

La aplicación de la escala fue auto contestable, no se reportaron observaciones y varió entre 15 y 30 minutos. El coeficiente alpha de Cronbach fue o.824, lo cual se considera aceptable, la prueba Kolmogorov-Smirnov no fue significativa ( $p=0.129$ ), se asume una curva con distribución normal de los datos, lo que significa que se cumple el postulado para realizar el análisis con estadística paramétrica. El promedio de la variable de adopción al rol materno fue de 78.9 DE \pm 0.9 . Las subescalas se comportaron de una manera similar, el microsistema tuvo un promedio de $64.5 \mathrm{DE} \pm$ 8.9; el meso-

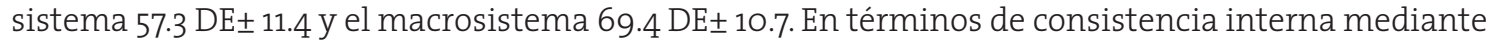
alpha de Cronbach, el microsistema tiene 0.803; mesosistema obtuvo 0.444 y macrosistema 0.351.

Se aplicó un análisis factorial exploratorio mediante el método de extracción de componentes principales, el índice de KMO fue de 0.644 , valor que se considera conveniente y el test de esfericidad de Bartlett (.oo1) con valor significativo, con lo que se demuestra que existe correlación estadísticamente significativa entre las variables con la ARM. Se determinaron tres componentes teóricos subyacentes del instrumento (microsistema, mesosistema y macrosistema), los cuales explican 36.2\% de la varianza total. Para mejorar la comprensión en la extracción de los datos y diferencia de cargas factoriales para cada reactivo, se realizó la rotación varimax, como se muestra en la tabla 2.

Tabla 2. Análisis Factorial Exploratorio con rotación varimax

\begin{tabular}{|c|c|c|c|c|c|c|c|c|c|}
\hline \multicolumn{10}{|c|}{ Varianza total explicada } \\
\hline \multirow[t]{2}{*}{ Factor } & \multicolumn{3}{|c|}{ Autovalores iniciales } & \multicolumn{3}{|c|}{$\begin{array}{l}\text { Sumas de las saturaciones } \\
\text { al cuadrado de la extracción }\end{array}$} & \multicolumn{3}{|c|}{$\begin{array}{l}\text { Suma de las saturaciones } \\
\text { al cuadrado de la rotación }\end{array}$} \\
\hline & Total & $\begin{array}{c}\% \text { de la } \\
\text { varianza }\end{array}$ & $\begin{array}{c}\% \\
\text { acumulado }\end{array}$ & Total & $\begin{array}{c}\% \text { de la } \\
\text { varianza }\end{array}$ & $\begin{array}{c}\% \\
\text { acumulado }\end{array}$ & Total & $\begin{array}{c}\% \text { de la } \\
\text { varianza }\end{array}$ & $\begin{array}{c}\% \\
\text { acumulado }\end{array}$ \\
\hline 1. & 10.162 & 19.925 & 19.925 & 10.162 & 19.925 & 19.925 & 7.824 & 15.341 & 15.341 \\
\hline 2. & 5.291 & 10.374 & 30.298 & 5.291 & 10.374 & 30.298 & 7.603 & 14.907 & 30.248 \\
\hline 3. & 3.019 & 5.920 & 36.218 & 3.019 & 5.920 & 36.218 & 3.045 & 5.971 & 36.218 \\
\hline
\end{tabular}

Método de extracción: Análisis de componentes principales 1. Microsistema; 2. Mesosistema; 3. Macrosistema

\begin{tabular}{cccc}
\multicolumn{4}{c}{ Matriz de Transformación de Factores } \\
\hline Factor & 1 & 2 & 3 \\
\hline 1 & .723 & .690 & .019 \\
\hline 2 & .685 & -.721 & .101 \\
\hline 3 & -.083 & .060 & .995 \\
\hline
\end{tabular}

Método de extracción: Análisis de componentes principales. Método de Rotación: Varimax con normalización de Kaiser 1. Microsistema; 2. Mesosistema; 3. Macrosistema 


\section{DISCUSIÓN}

La discusión se abordará a partir de las etapas establecidas en la metodología y sus particularidades.

Primera etapa. La adecuación semántica ${ }^{18}$ a ocho de los ítems originales se enfocó en sustituir lactancia, dar(le) de lactar por las palabras pecho, dar(le) pecho; ya que es un término común de expresión en el contexto sociocultural mexicano ${ }^{15}$ para referirse a la lactancia materna, situación que en una adolescente puede ser mejor comprendida. El término pecho también es utilizado comúnmente por profesionales de la salud, principalmente de enfermería, quienes fungen como educadores sobre el tema en madres primerizas.

Segunda etapa. Respecto a la validación por jueces ${ }^{13,16,17}$, el IVXI, el CV y el IVC representan la validez interna. Los resultados obtenidos muestran que el contenido de la escala es correcto de acuerdo con la definición del concepto representado en los tres factores del sistema, tal cual lo menciona Mercer: Microsistema, Mesosistema y Macrosistema con un total de diez dimensiones ${ }^{6}$.

Estos resultados son similares a los encontrados por Garrido y Marchán ${ }^{12}$ así como por Vargas et al. ${ }^{10}$, quienes también realizaron el análisis de la E-ARM ${ }^{12}$ a partir de diez jueces expertos en la materia de estudio; cuyos resultados prueban la claridad, coherencia y relevancia con respecto al concepto que se dice medir ${ }^{16}$. Desde luego, los ítems que corresponden a diferentes dimensiones de la escala están fundamentados en una base teórica sólida, congruente y ampliamente probada en diversos contextos, razones que son fundamentales y suficientes para mostrar la validez de la escala.

Tercera etapa. En esta etapa no se sugirieron cambios a la E-ARM por parte de los sujetos de estudio debido -principalmente- a las modificaciones realizadas con anterioridad por los autores (adecuación semántica y cultural) ${ }^{15} \mathrm{y}$ por los jueces ${ }^{16}$, quienes evaluaron la validez de los ítems (estimación cuantitativa y cualitativa), ademas aportaron directamente a la estructura de éstos, de tal manera que fue considerada la versión final de la adaptación de la escala, objetivo de este trabajo.

Cuarta etapa. La versión aplicada a 90 adolescentes no tuvo observaciones. Las propiedades psicométricas de la escala mostraron ser sólidas en el contexto cultural mexicano, referidas por la distribución normal de los datos y la consistencia interna obtenida; resultados similares a los de Garrido y Marchán ${ }^{12}$ y Vargas et al..10. Sin embargo, ambos trabajos reportan valores ligeramente más elevados que en el presente artículo, esto pudiera deberse a que la aplicación del instrumento, a diferencia de los estudios ya mencionados, se realizó en el contexto hospitalario, donde existe un sinfín de estímulos externos que pudieron haber interferido en el momento de responder la E-ARM ${ }^{12}$

Por ejemplo, se encontró que la mayoría de las adolescentes cursaban la etapa de adolescencia tardía, tenían un nivel educativo básico incompleto y se dedicaban a las labores del hogar, datos similares a los reportados por Garrido y Marchán ${ }^{12}$ en Perú. No obstante, los resultados difieren con los encontrados por Vargas et al..$^{10}$ en Colombia, en donde las mujeres del estudio se encuentran en el inicio de la adultez y reportan una muestra en su mayoría universitaria, lo que se explica a partir de las variaciones en la ARM. Teóricamente, la ARM es un proceso gradual de adaptación influido por las condiciones sociales y educativas de la madre, las cuales repercuten para fomentar y mejorar el proceso o tal vez podrían fungir de forma contraria. Además, las funciones ejecutivas en adolescentes mayores de edad están más desarrolladas por los mismos procesos de crecimiento y desarrollo, tales como la toma de decisiones y fijación de metas ${ }^{5,6}$.

De acuerdo con Mercer 6 , el proceso de ARM se produce de forma efectiva incluyendo las actividades de cuidado del bebé hasta los procesos cognitivo-afectivos, los cuales son independientes del evento obstétrico, ya que el rol materno abarca aspectos mucho más complejos que el medio o canal por el cual se haya presentado el nacimiento. Esta situación coincide con los datos obtenidos, donde predominó la cesárea como medio para el nacimiento y es similar a lo presentado por Vargas et al. ${ }^{10}$. 
Respecto a las adecuaciones que se realizaron, al no considerar los valores crudos de la escala sino la transformación de la variable en índice de cero a cien, se le dio a la escala un carácter más sencillo de comprensión sobre el nivel de ARM. Además, se realizó una propuesta de puntos de corte cada 25 puntos para identificar el nivel de ARM, a diferencia de las escalas de Garrido y Marchán ${ }^{12}$ y Vargas et $a .^{10}$. Se considera que este aspecto facilitará la interpretación de los resultados al ser utilizado para manipular la variable a través de intervenciones ${ }^{19,20}$ educativas en salud y no solo ver el efecto a través de los promedios aritméticos y la significancia estadística, situación que le da un sentido más pragmático al aplicarlo en condiciones clínicas, lo que fortalecerá los cuidados de enfermería de forma individual a cada una de las nuevas mamás. Con el fin de conservar la orientación en el puntaje de los ítems, se han señalado los evaluados en sentido inverso para disminuir el error susceptible al momento de responder el instrumento, disminuyendo el efecto de techo-piso y obteniendo una variabilidad para reforzar la validez de la escala.

La nueva versión de la E-ARM (Tabla 3) en el contexto mexicano refuerza a la original de Garrido y Marchán ${ }^{12}$, si bien los procesos de validación de los instrumentos pueden darse por varias técni$\operatorname{cas}^{20,21}$, la escala del presente artículo complementa a la utilizada por los autores originales ${ }^{12}$ y los autores colombianos ${ }^{10}$. Se recomendaría probar la sensibilidad de la escala a través de intervenciones de apoyo educativo con diseños clínicos para reforzar en gran medida el impacto de los cuidados de enfermería en este grupo de mujeres.

\section{Tabla 3. Versión final del instrumento}

ESCALA DE ADOPCIÓN DEL ROL MATERNO (Garrido y Marchan, 2011)

Adaptación al contexto mexicano: Santos-Díaz María de la Luz, Pérez-Calderón Dulce María, LozadaPerezmitre Erika, Ramírez-Girón Natalia; Landeros-Olvera Erick (2020).

INSTRUCCIONES: A continuación encontrará una lista de afirmaciones respecto a su experiencia como madre. Deberá elegir, marcando con un aspa (X), la opción que más se asemeje a su forma de pensar, actuar y sentir. No hay respuestas buenas ni malas. Las opciones de respuesta son las siguientes: TD=Totalmente en desacuerdo. $\mathrm{D}=$ Desacuerdo. A=De Acuerdo. TA=Totalmente de Acuerdo.

\begin{tabular}{|c|c|c|c|c|c|}
\hline & & TD & D & A & TA \\
\hline 1 & Prefiero no darle pecho a mi bebé para cuidar la apariencia de mis senos. & & & & \\
\hline 2 & Imito los balbuceos y sonidos que hace mi bebé. & & & & \\
\hline 3 & Estoy dispuesta a cambiar el pañal a mi bebé cada vez que sea necesario. & & & & \\
\hline 4 & Leo información referente a los cuidados y temas relacionados con mi bebé. & & & & \\
\hline 5 & Me cuesta aceptar a mi bebé. & & & & \\
\hline 6 & Cuando mi bebé llora dejo lo que estoy haciendo y voy a atenderlo. & & & & \\
\hline 7 & $\begin{array}{l}\text { Pienso que darle pecho a mi bebé debería ser sustituido por la alimentación en } \\
\text { biberón. }\end{array}$ & & & & \\
\hline 8 & Me cuesta decir que soy madre. & & & & \\
\hline 9 & Me siento capaz de salir adelante con mi bebé. & & & & \\
\hline 10 & Mi familia está contenta con el nacimiento de mi bebé. & & & & \\
\hline 11 & Le doy objetos a mi bebé para que los pueda tocar y aprenda a manipularlos. & & & & \\
\hline 12 & $\begin{array}{l}\text { Mi pareja y yo (o la persona que me ayuda) siempre estamos poniéndonos al } \\
\text { tanto de las cosas que le pasan al bebé. }\end{array}$ & & & & \\
\hline 13 & $\begin{array}{l}\text { Cuando mi bebé llora le hablo con voz muy baja y susurrando palabras } \\
\text { tranquilizadoras. }\end{array}$ & & & & \\
\hline
\end{tabular}


14 Prefiero ser yo quien se encargue del baño de mi bebé.

15 Cuando estaba embarazada pensaba con entusiasmo en el momento en que tendría a mi bebé en brazos dándole pecho.

16 Pienso que el control del niño sano no es necesario para mi bebé.

17 El personal de salud me ha enseñado cuidados que debo tener con mi bebé.

18 Cuando le hablo a mi bebé lo hago con palabras cariñosas.

19 Cuando juego con mi hijo me gusta que él aprenda algo nuevo.

20 Cuando doy pecho a mi bebé me gusta hablarle.

21 Me gusta mirar detenidamente a mi bebé para conocerlo mejor.

22 Me hubiera gustado que mi bebé fuera del sexo opuesto al que nació.

23 El momento de juego con mi bebé es indispensable en mi rutina diaria.

24 La salud de mi bebé para mí es muy importante.

25 Para mí, dar el pecho a mi bebé es más una obligación que una actividad agradable.

26 Cuando mi bebé responde a mis llamados, caricias u órdenes, yo lo abrazo o felicito.

27 Me agrada masajear la espalda de mi bebé con mucha delicadeza.

28 Mi pareja (o la persona que me ayuda) me recuerda cuando mi bebé debe recibir sus vacunas y asistir al control del niño sano.

29 Prefiero que alguien de mi familia se encargue de alimentar a mi bebé.

30 Mientras doy el pecho a mi bebé me gusta acariciarlo.

31 Cuando le hago cosquillas a mi bebé observo una respuesta de alegría en él.

32 Cuando voy a un lugar público hago valer mis beneficios por ser madre y estar con mi hijo en brazos.

33 Aunque tenga muchas actividades que realizar, siempre me doy tiempo para cuidar a mi bebé.

34 Constantemente me informo por redes sociales de cómo cuidar a mi bebé.

35 Llevo a vacunar a mi bebé en la fecha programada.

36 Sé que cuento con el apoyo de mi familia para el cuidado de mi bebé.

37 Prefiero que mi hijo tome biberón a darle el pecho.

38 Trato de que mi bebé acaricie mi rostro.

39 Tengo la seguridad de que seré una buena madre.

40 Mi pareja (o la persona que me ayuda) y yo compartimos los cuidados del bebé.

41 Cuido que mi bebé utilice ropa adecuada a la estación del año para evitar que se enferme o le salgan ronchitas por el sudor.

42 Creo que dar el pecho a mi hijo nos une emocionalmente.

43 Me alegra mucho que mi hijo haya nacido sano.

44 Cada vez que mi bebé llora me preocupo por saber qué es lo que necesita.

45 La forma en que crio a mi bebé es muy parecida a como me criaron a mí.

46 Mi familia me aconseja sobre la crianza de mi bebé.

47 Me preocupo porque alrededor de mi bebé no existan objetos que puedan causarle daño.

48 Trato de ocultar que soy madre porque me avergüenza.

49 Creo que otro familiar cuida a mi bebé mejor que yo.

50 Terceras personas se encargan de la crianza de mi bebé. 
51 Si voy por la calle y alguien estornuda cerca, lo primero que hago es cubrir a mi bebé.

52 Mi pareja (o la persona que me ayuda) es capaz de hacerse cargo de mi bebé cuando yo no lo puedo hacer.

53 Practico con mi bebé ejercicios que le puedan ayudar a su desarrollo.

54 Diariamente mi pareja (o la persona que me ayuda) busca saber cómo está el bebé y coordinarnos acerca de cómo satisfacer sus necesidades.

Asimismo, se encontraron algunos aspectos de mejora para la E-ARM de Garrido y Marchán ${ }^{12}$, ya que en la estructura factorial de la escala original se cuentan con 56 ítems; sin embargo, en la versión final del instrumento sólo se reportan 54 y no se menciona la eliminación de los faltantes en las diferentes etapas del proceso (ítem 55. Las cosas que aprendí sobre el cuidado de mi bebé me las enseñaron las personas que me criaron; item 56. Tengo en casa medicamentos necesarios para lo que requiere mi bebé en caso de que enferme). De igual forma, en la versión final del instrumento se incluye un ítem que no se menciona dentro del análisis factorial (ítem 37. Prefiero que mi hijo tome biberón a darle de lactar). No obstante, estas consideraciones no se mencionan en el trabajo realizado en la muestra colombiana ${ }^{10}$, dado que los autores sí reportan la totalidad de los ítems de la escala.

\section{CONCLUSIÓN}

Se adaptó la Escala de ARM de Garrido y Marchan para madres adolescentes mexicanas, la cual se consideró válida y confiable. Es necesario que la escala sea probada en otros contextos socioculturales dentro del territorio mexicano, con muestras más grandes y representativas, con diseños longitudinales que permitan comprender la variación del rol materno a partir de las diferentes etapas del puerperio y durante el crecimiento de los bebés.

De igual forma, es fundamental probar la sensibilidad del concepto al manipular la variable con intervenciones educativas de enfermería a través de ensayos clínicos controlados. Los resultados se deben tomar con reserva, pues a pesar de que la escala fue adaptada al contexto sociocultural mexicano, las regiones geográficas del país contienen variabilidad y situaciones específicas que deben de considerarse.

\section{RESPONSABILIDADES ÉTICAS}

Protección de personas y animales. Los autores declaran no haber realizado experimentos en seres humanos ni en animales para el desarrollo de esta investigación.

Conflicto de intereses. Los autores declaran no tener conflicto de intereses.

Financiamiento. Ninguno.

\section{REFERENCIAS}

1. Development Centre-Organization for Economic Co-operation and Development/Social Institutions and Gender Index. Latin America and the Caribbean: SIGI Regional Report. Paris: OECD; 2017. https://bit.ly/3v8sJWA

2. Azevedo JP, Favara M, Haddock SE, López-Calva LF, Müller M, Perova E. Embarazo adolescente y oportunidades en América Latina y el Caribe: sobre maternidad temprana, pobreza y logros económicos. Washington D.C.: Banco Internacional de Reconstrucción y Fomento/Banco Mundial; 2012. https://bit.ly/zeW4IfU 
3. Fondo de las Naciones Unidas para la Infancia. Estado Mundial de la Infancia 2011: la adolescencia, una época de oportunidades. Nueva York: UNICEF; 2011. https://uni.cf/33PoZdR

4. Instituto Nacional de Mujeres. Estrategia nacional para la prevención del embarazo en adolescentes. México: Gobierno de México; 2020. https://bit.ly/3foLsa3

5. Alamo N, Krause M, Pérez, JC, Aracena M. Impacto de la salud psicosocial de la madre adolescente en la relación con el niño/a y su desarrollo. Rev. argent. clín. psicol. 2017; 26(3): 332-46.

https://doi.org/10.24205/03276716.2017.1022

6. Mercer RT. Adopción del rol materno-convertirse en madre. En: Raile-Alligood M, MarrinerTomey A. Modelos y teorías en enfermería. $7^{\mathrm{a}}$ ed. España: Elsevier; 2011.

7. Nongnut-Boonyoung E, Chunuan S. Evaluation of the Psychometric Properties of Self-Efficacy in Performing Maternal Role for First Time Pregnant Adolescents in Indonesia. Songklanagarind J Nurs. 2020; 4O(2): 10-23. https://bit.ly/3uVMpgB

8. Vargas-Vásquez AN, Pardo-Torres MP. Validez y consistencia interna del instrument Vínculo entre padres e hijos neonates. Enferm. glob. 2020; 19(59): 255-70.

https://dx.doi.org/10.6018/eglobal.403721

9. Nieto L, Lara MA, Navarrete L. Prenatal predictors of maternal attachment and their association with postpartum depressive symptoms in Mexican women at risk of depression. Matern Child Health J. 2017; 21: 1250-59. https://doi.org/10.1007/s10995-016-2223-6

10. Vargas-Porras C, Roa-Díaz ZM, Hernández-Hincapié HG, Ferré-Grau C, De Molina-Fernández MI. Adaptación cultural, validez y confiabilidad de la escala de adopción del rol materno en Colombia. Cienc. innov. salud. 2020; e70: 1-18. https://doi.org/10.17081/innosa.70

11. Lascano-Paredes DC. La adaptabilidad al rol materno y su influencia en los síntomas ansiosos depresivos en mujeres en la etapa postneonatal. Caso: Centro de Salud Ingahurco Ambato área 1. [Tesis]. Ambato, Ecuador: Pontificia Universidad Católica del Ecuador. 2018. https://bit.ly/33Ro8fN

12. Garrido-Hidalgo MC, Marchán-Coronado M. Adopción del rol materno en madres adolescentes primerizas según grupo de convivencia. Rev. Psicol. 2011; 13(1): 11-28. https://bit.ly/3uTqu9H

13. Waltz CF, Strickland O, Lenz ER. Measurement in nursing and health research. New York: Springer publishing company; 2010.

14. López-Fernández R, Avello-Martínez R, Palmero-Urquiza DE, Sánchez-Gálvez S, Quintana-Álvarez M. Validación de instrumentos como garantía de la credibilidad en las investigaciones científicas. Rev. cuba. med. mil. 2019; 48(2 sup): 441-50. https://bit.ly/2S1LCvS

15. Ayala-Peréz T. Comunicación y comunicaciones: ¿un problema semántico o cultural?. Contextos. 2017; (3): 13-37. https://bit.ly/3fd7H3n

16. Galicia-Alarcón LA, Balderrama-Trápaga JA, Edel-Navarro R. Validez de contenido por juicio de expertos: propuesta de una herramienta virtual. Apert. 2017; 9(2): 42-53.

http://dx.doi.org/10.32870/Ap.vgn2.993

17. Juárez-Hernández LG, Tobón S. Análisis de los elementos implícitos en la validación de contenido de un instrumento de investigación. Espacios. 2018; 39(53): 23-9. https://bit.ly/3hzazcT

18. Abraham S, Kiefer F. A theory of structural semantics. Alemania: Walter de Gruyter GmbH \& Co. KG: 2018.

19. Meighan M. Mercer RT: Maternal role attainment-becoming a mother. En: Raile Alligood M. Nursing Theorists and their work. $9^{e}$ ed. Missouri, EE.UU.: Elsevier; 2017.

20. Nagalakshmi E. Application of nursing theories in pediatric nursing. J Pediatr Nurs. 2017; 5(1): 2532. https://bit.ly/2T1R7LF

21. Swartz ME, Krull IS. Analytical method development and validation. New York: CRC Press; 2018. 